\title{
The effect of distracting stimuli on the runway performance of limbic damaged rats'
}

\author{
ALFRED C. RAPHELSON, FLINT COLLEGE, UNIVERSITY OF MICHIGAN \\ ROBERT L. ISAACSON AND ROBERT J. DOUGLAS ${ }^{2}$, UNIVERSITY OF MICHIGAN
}

\begin{abstract}
Abstraet
The introduction of novel visual stimuli failed to disturb the performance of animals with extensive bilateral destruction of the hippocampus in a linear runway. Animals with destruction restricted to the dorsal hippocampus and animals with damage of the septal area were less distracted on first presentation of the distracting stimuli but recovered former running speeds more quickly than control groups. Novel tactual stimuli failed to affect the performance of animals in all groups.
\end{abstract}

Effects of limbic destruction have often been interpreted as suggesting that these structures are involved in the inhibition of certain kinds of responses. For example, hippocampectomized rats have been reported to take longer to extinguish a conditioned avoidance response (Isaacson, Douglas, \& Moore, 1961), runway responses (Niki, 1962; Jarrard, Isaacson, \& Wickelgren, 1964; Jarrard \& Isaacson, 1965), and perform poorly when shifted to a differential reinforcement schedule (DRL 20) after earlier training on a continuous reinforcement schedule (Clark \& Isaacson, 1965).

An alternative to the response inhibition explanation of hippocampal and other limbic destruction may be that such animals are deficient in abilities required to attend to or orient toward stimuli other than the ones to which they had been trained. In support of this argument, Wickelgren \& Isaacson (1963) found that the sudden introduction of irrelevant stimuli into a runway in which a well-established running response was being performed did not affect the latency of hippocampectomized rats but significantly increased the latency of their control Ss. There is related evidence that changes in "orienting response" may follow hippocampal destruction (Grastyan \& Karmos, 1961).

The present study attempts to clarify the role of limbic system structures in the behavior of animals following the introduction of novel stimuli. The earlier work (Wickelgren \& Isaacson, 1963) utilized a distracting stimulus having both visual and tactual properties and Ss with only one type of limbic damage (radical bilateral hippocampectomy)。In this study, visual and tactual distractions were presented separately and the following three experimental groups with lesions of the limbic system were used: (1) radical bilateral hippocampal destruction, (2) partial bilateral destruction of dorsal hippocampus, and (3) bilateral septal lesions.

\section{Method}

Thirty naive male hooded rats, approximately 90 days old, were used as Ss and maintained throughout the experiment on a $23 \mathrm{hr}$. food deprivation schedule.

The apparatus was a straight, black runway, 96 in by 5 in by 5 in. One door was located 12 in down the runway incorporating a "start box" and another door was located 66 in farther down marking the beginning of an 18 in "goal box." A partition was set 2-1/2 in in front of the back wall behind which was placed a glass food dish. One $97 \mathrm{mg}$ Noyes food pellet served as a reward on each trial.

Prior to surgery all animals received 50 rewarded trials in blocks of five trials a day with a minimal $5 \mathrm{~min}$. intertrial interval. Running speed was obtained on all trials. At the end of this preoperative training, the Ss were divided into six groups of five each matched on average running speed for the last day of training.

Four groups of animals were prepared by surgical techniques which were made by aspiration of neural tissue. These were: Radical Bilateral Hippocampal group. Cortical tissue overlying the hippocampus plus dorsal, medial and ventral portions of the hippocampus was removed. Partial Bilateral Hippocampal group. Only the dorsal portion of the hippocampus, plus overlying neocortical tissue, was removed. Bilateral Cortical Control group. The neocortex overlying the hippocampus was removed and the lesion extended so that more tissue was removed than in the hippocampally lesioned animals. The hippocampus itself was not destroyed. Ablation Sham Control group. This group was prepared for surgery, but the brain was not damaged. Two groups were prepared for electrolytic lesions while held in a Johnson stereotaxic instrument. These were: Bilateral Septal group. Lesions were produced by passing 1.8 ma anodal current for $45 \mathrm{sec}$. through an electrode introduced according to the following coordinates: $1.8 \mathrm{~mm}$ anterior to bregma; $3.0 \mathrm{~mm}$ lateral $; 4.5 \mathrm{~mm}$ below the surface of the brain at an angle of $25^{\circ}$ toward the midline. Electrolytic Sham Control group. These animals were prepared like the first group but no current was passed through the electrode. Following a two week recovery period, the Ss received 45 retraining trials under the preoperative training conditions. On the day following the start of retraining, the animals were given 10 trials according to the following schedule: Trials 1 and 2 (N1 and N2) were the same as previous training trials; Trials 3 and 4 (V1 and V2) were given with two pieces of white poster board, 22 in by $4 \mathrm{in}$, attached to the sides of the runway near the "start box" door. These were the visual distractors; Trials 5 and 6 (N3 and N4) were standard trials like N1 and N2; Trials 7 and 8 (T1 and T2) were given with a flat metal rectangle, 5 in by $4 \mathrm{in}$, covered with fine sandpaper placed midway in the runway. This was the tactual distractor. Trials 9 and 10 (N5 and N6) were similar to $\mathrm{N} 1, \mathrm{~N} 2, \mathrm{~N} 3$, and N4.

After the experiment, the animals were sacrificed, their brains removed and embedded in paraffin. Sections were made at $20 \mu$ and stained with thionin or a luxol fast blue-cresyl violet counterstain. Examination of the sections for animals in the Radical Hippocampal group showed the entire dorsal and medial portions of the hippocampus were destroyed. In some animals the destruction reached into the ventral aspects below the rhinal fissure. All animals in the Septal group received extensive damage to that structure and in some cases there was extraneous damage to adjacent areas. However, despite this extraneous damage, all members of this group exhibited amazing homogeneity of behavior. Animals in the Partial Hippocampal groups were found to have suffered destruction of only the dorsal hippocampus plus overlying neocortical tissue. 


\section{Results}

Two Ss in the Cortical Control group were found to have damage to the dorsal hippocampus, unilaterally in one $\mathrm{S}$ and bilaterally in the other. Both Ss showed behavior more typical of hippocampally damaged animals than of cortically damaged animals.

The effect of the distracting visual stimuli was determined by expressing the running speed of the distraction trials (V1, V2, T1, T2) as a percentage of the preceding neutral trials (N2 or N4). The tactual distractors produced no significant differences among the groups. Figure 1 shows the results for the trials in which the visual distracting stimuli were presented.

All groups, except the Radical Hippocampal group, decreased their running speed on the first visual distraction trial (VI). This latter group showed an increase in speed that was significantly greater than the speed of the other five groups $(p=.05)$. On the second visual distraction trial (V2) all groups with limbic damage showed significant increases $(p=.01)$ in running speed over the immediately preceding trial (VI). The Radical Hippocampal group continued its increase in speed and the Partial Hippocampal and Septal groups showed significant recoveries from their previous distractibility. Although all three control groups showed some recovery on the second visual distraction trial (V2), only the Ablation Sham group's change reached significance $(p=.05)$. The combined limbic damage versus control group effect is shown in the lower part of Fig. 1. The lesions effect in this combined comparison was significant $(\mathrm{F}=4.29, \mathrm{p}=.05)$.

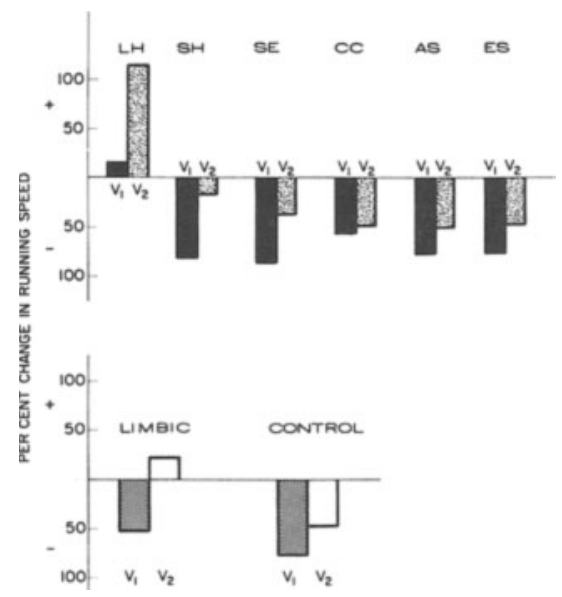

Fig. 1. Upper: Per cent change in running speeds of the six groups of animals on trials when visual distractors were present expressed as differences from running speeds on earlier trials (N1 and N2). Lower: The same date combined into groups suffering limbic damage (LH, SH, SE) and control groups (CC, AS, ES).
The most significant finding in this study was that only the animals with extensive hippocampal destruction showed the complete lack of distractibility reported earlier. The other limbically damaged groups showed a quick recovery from distractibility but were similar to the control groups in their initial performance.

Animals with partial destruction of the hippocampus and the animals with destruction of the septal area showed greater recovery from distraction. Since these latter groups did show initial distractibility, it is not possible to apply the orienting response dysfunction hypothesis as a general explanation for the consequence of all limbic damage. Recently, Snyder \& Isaacson (1965) have shown that lesions restricted to the dorsal aspects of the hippocampus fail to produce much debility when tested in two kinds of passive avoidance problems. The fact that septal and partial hippocampal damage did produce significantly greater recovery from the distraction, even though distraction did appear, suggests that different functions may be involved in these different parts of the limbic system.

\section{References}

Clark, C. V., \& Isaacson, R. L. Effect of bilateral hippocampal ablation on DRL performance. J. comp. physiol. Psychol., 1965, $59,137-140$.

Grastyan, E., \& Karmos, G. The influence of hippocampal lesions on simple and delayed instrumental conditioned reflexes. In J. Cadillac (Ed.), Physiologie de l' Hippocampe. colloq. intern. du CNRS. Montpellier, 1961.

Isaacson, R. L., Douglas, R. J., \& Moore, R. Y. The effect of radical hippocampal ablation of acquisition of avoidance responses. J. comp. physiol. Psychol., 1961, 54, 625-628.

Jarrard, L. E., \& Isaacson, R. L. Hippocampal ablation in rats: effects on intertrial interval. Nature, 1965, 207, 109-110.

Jarrard, L. E., Isaacson, R. L., \& Wickelgren, W. O. Effects of hippocampal ablation and intertrial interval on runway acquisition and extinction. J. comp. physiol. Psychol., 1964, 57, 442-444.

Niki, H. The effects of hippocampal ablation on the behavior of the rat. Jap. psychol. Res., 1962, 4, 139-153.

Raphelson, A. C., Isaacson, R. L., \& Douglas, R. J. The effect of limbic damage on the retention and performance of a runway response. In preparation, 1965.

Snyder, D. R., \& Isaacson, R. L. The effects of large and small bilateral hippocampal lesions on two types of passive avoidance responses. Psychol. Rep., 1965, 16, 1277-1290.

Wickelgren, W. O., \& Isaacson, R. L. Effect of the introduction of an irrelevant stimulus upon runway performance of the hippocampectomized rat. Nature, 1963, 200, 48-50.

\section{Notes}

1. The research was carried out while the first author was a National Science Foundation Research Participation Frogram for College Teachers of Psychology participant at the University of Michigan in 1963. Support for certain aspects of the research was from Grant DA-MD-49-193-64-G120 from the Surgeon General, U. S. Army to Robert L. Isaacson.

2. Dr. Douglas' present address is: Department of Psychiatry, R-321, Stanford University School of Medicine, Stanford Medical Center, Palo Alto, California, 94304. 